tertational Electronic Journal of Algebra

VOLUME 22 (2017) 187-201

DOI: $10.24330 /$ ieja.325944

\title{
BLOCK DECOMPOSITION FOR MODULES
}

\author{
H. Khabazian \\ Received: 14 April 2017; Revised: 9 June 2017 \\ Communicated by Abdullah Harmancı
}

\begin{abstract}
Block decomposition for rings has been introduced and shown to be unique in the literature (see [T. Y. Lam, Graduate Texts in Mathematics, 131, Springer-Verlag, New York, 1991]). Applying annihilator submodules, we extend this definition to modules and show that every module $M$ has a unique block decomposition $M=\bigoplus_{i=1}^{n} M_{i}$ where each $M_{i}$ is an annihilator submodule. We also show that the block decomposition for any ring $R$ and the block decomposition for the module $R_{R}$, are identical. Block decomposition provides us with a decomposition for $\operatorname{End}(M)$ because $\operatorname{End}(M) \cong \prod_{i=1}^{n} \operatorname{End}\left(M_{i}\right)$.
\end{abstract}

Mathematics Subject Classification (2010): 16D70, 16D50, 16D80

Keywords: Annihilator, block orthogonal, block decomposable, $\mathcal{G}$-organized

\section{Introduction}

Any ring with unit satisfying some finiteness conditions on ideals, is isomorphic to the direct product of a finite family of indecomposable rings and this representation is unique $[4,2.22]$, in the following sense. Let each $R_{i}$ and each $S_{j}$ be an indecomposable ring. If there is an isomorphism $\Delta: \prod_{i=1}^{n} R_{i} \longrightarrow \prod_{j=1}^{k} S_{j}$, then $k=n$, and, after a re indexing, there is isomorphisms $\Delta_{i}: R_{i} \longrightarrow S_{i}$ for $1 \leq i \leq n$ such that $\Delta=\prod_{i=1}^{n} \Delta_{i}$. But the decomposition structure for modules is not unique. Even if decomposition structure is unique for certain modules [4, 19.22, Krull-Schmidt Theorem], it is not unique in the above sense. The reason is that viewing a ring as a module on itself, the class of submodules is not similar to the class of ideals.

Applying annihilator submodules and adjusted techniques, we will be able to introduce a ring type decomposition for modules. Also, with the same techniques, we show that any left or right faithful ring, not necessary having unit and satisfying even weaker finiteness conditions in [4, 2.22], is decomposable.

In Section 1, we outline a general theory in additive group format so that a large part of the proofs of some theorems would become special case of a theory that is useful in this context and is of intrinsic interest by itself. In Section 2, 
we introduce annihilator submodules, then standard facts are collected, some basic properties are developed and we apply Proposition 2.16 to derive the existence of the block decomposition for rings and modules. In Section 3, we show that the block decomposition for a ring $R$ and block decomposition for $R_{R}$ are identical.

In this paper, for any set $S$ of subgroups of an additive group, we set $\Sigma(S)=$ $\sum_{I \in S} I$ and $S$ is said to be independent if $\sum_{I \in S} I$ is a direct sum. Also, we use the notation $\bigoplus(S)$ instead of $\Sigma(S)$ to indicate that $S$ is independent.

For any class $\mathcal{C}$ of subgroups, a $\mathcal{C}$-subgroup means a subgroup from the class $\mathcal{C}$, the class of minimal $\mathcal{C}$-subgroups is denoted by $\mathcal{C}^{\text {mn }}$ and the class of maximal $\mathcal{C}$-subgroups is denoted by $\mathcal{C}^{\mathrm{mx}}$.

For classes $\mathcal{C}$ and $\mathcal{F}$ of subgroups, the class of subgroups which are a $\mathcal{C}$-subgroup and an $\mathcal{F}$-subgroup is denoted by $\mathcal{C} \cap \mathcal{F}$.

For an additive group $M$, the set of $\mathcal{C}$-subgroups of $M$ is denoted by $\langle\mathcal{C}: M\rangle$, $M$ is called $\mathcal{C}$-semisimple if $M=\Sigma\left\langle\mathcal{C}^{\mathrm{mn}}: M\right\rangle, M$ is called $\mathcal{C}$-sum if the sum of any two $\mathcal{C}$-subgroups is a $\mathcal{C}$-subgroup, $M$ is called generalized $\mathcal{C}$-sum if the sum of any set of $\mathcal{C}$-subgroups is a $\mathcal{C}$-subgroup, $M$ is called $\mathcal{C}$-d.sum if the direct sum of any two $\mathcal{C}$-subgroups is a $\mathcal{C}$-subgroup, $M$ is called generalized $\mathcal{C}$-d.sum if the sum of any independent set of $\mathcal{C}$-subgroups is a $\mathcal{C}$-subgroup, $M$ is called $\mathcal{C}$-intersection if the intersection of any two $\mathcal{C}$-subgroups is a $\mathcal{C}$-subgroup, $M$ is called generalized $\mathcal{C}$-intersection if the intersection of any set of $\mathcal{C}$-subgroups is a $\mathcal{C}$-subgroup, $M$ is called $\mathcal{C}$-Artinian if $M$ satisfies the descending chain condition on $\mathcal{C}$-subgroups, in other words, if its $\mathcal{C}$-subgroups satisfy DCC, $M$ is called $\mathcal{C}$-Noetherian if $M$ satisfies the ascending chain condition on $\mathcal{C}$-subgroups, in other words, if its $\mathcal{C}$-subgroups satisfy ACC, $M$ is called $\mathcal{C}$-mini if every nonzero $\mathcal{C}$-subgroup contains a minimal $\mathcal{C}$-subgroup of $M, M$ is called $\mathcal{C}$-maxi if every proper $\mathcal{C}$-subgroup is contained in a maximal $\mathcal{C}$-subgroup of $M, M$ is called $\mathcal{C}$-ind.finite if every independent set of $\mathcal{C}$-subgroups is finite.

For an additive group $M$ and $K \subseteq M$, the set of $\mathcal{C}$-subgroups of $M$ containing $K$ is denoted by $\langle\mathcal{C} \supseteq K\rangle$, the set of $\mathcal{C}$-subgroups of $M$ contained in $K$ is denoted by $\langle\mathcal{C} \subseteq K\rangle$, and the set of $\mathcal{C}$-subgroups of $M$ not contained in $K$ is denoted by $\langle\mathcal{C} \nsubseteq K\rangle$.

For any additive groups $U, V$ and $W$, any $X \subseteq U, Z \subseteq W$ and multiplication $U \times V \longrightarrow W$, we set $(Z: X)=\{v \in V \mid X v \subseteq Z\}, \operatorname{ann}_{V}(X)=\{v \in V \mid X v=0\}$ and we say that $X$ is $V$-faithful if $\operatorname{ann}_{V}(X)=0$. For the case $V \times V \longrightarrow W$, we set $(Z: X)_{\mathrm{r}}=\{v \in V \mid X v \subseteq Z\}, \operatorname{ann}_{\mathrm{r}}(X)=\{v \in V \mid X v=0\}$ and we say $X$ is right faithful if $\operatorname{ann}_{\mathrm{r}}(X)=0$. 
For any family $S$ of subsets of a set, we set $\operatorname{Int}(S)=\bigcap_{I \in S} I$ and $\operatorname{Un}(S)=\bigcup_{I \in S} I$. In the context, $\mathcal{C}$ and $\mathcal{G}$ designate arbitrary classes of subgroups.

\section{Preliminaries}

Definition 2.1. Let $M$ be an additive group and $K$ be a subgroup.

(1) We say that $K$ is $\mathcal{C}$-summand if there is a $\mathcal{C}$-subgroup $J$ such that $M=$ $K \oplus J$.

(2) We say that $K$ is $\mathcal{C}$-indecomposable if for any $\mathcal{C}$-subgroups $I$ and $J, K=$ $I \oplus J$ implies $I=0$ or $J=0$.

(3) We say that $K$ is $\mathcal{C}$-uniform if $K \neq 0$ and for any $\mathcal{C}$-subgroups $I, J \subseteq K$, $I \cap J=0$ implies $I=0$ or $J=0$.

Also, the class of $\mathcal{C}$-summand subgroups is denoted by $\mathcal{C}^{\oplus}$. Recall that according to the above notifications, $\mathcal{C} \cap \mathcal{C}^{\oplus}$ is the class of $\mathcal{C}$-summand $\mathcal{C}$-subgroups, $\left(\mathcal{C} \cap \mathcal{C}^{\oplus}\right)^{\mathrm{mn}}$ is the class of minimal $\mathcal{C}$-summand $\mathcal{C}$-subgroups, and $\Sigma\left\langle\left(\mathcal{C} \cap \mathcal{C}^{\oplus}\right)^{\mathrm{mn}}: M\right\rangle$ is the sum of the minimal $\mathcal{C}$-summand $\mathcal{C}$-subgroups of $M$.

Definition 2.2. Let $M$ be an additive group. A map $\star:\langle\mathcal{G}: M\rangle \longrightarrow\langle\mathcal{G}: M\rangle$ is called $\mathcal{G}$-organizer if

(1) For any $\mathcal{G}$-subgroup $K, K \cap K^{\star}=0$ and $K^{\star \star}=K$.

(2) For any $\mathcal{G}$-subgroups $J$ and $K, K \cap J=0$ implies $J \subseteq K^{\star}$.

Also, $M$ is called $\mathcal{G}$-organized if it has a $\mathcal{G}$-organizer map.

If $\star$ is a $\mathcal{G}$-organizer map, then for any $\mathcal{G}$-subgroups $J$ and $K, K \subseteq J$ implies $J^{\star} \subseteq K^{\star}$ because we have $K \cap J^{\star}=0$.

Lemma 2.3. Let $M$ be an additive group and $K$ be a nonzero subgroup. If every independent set of $\mathcal{C}$-subgroups contained in $K$ is finite, then $K$ contains a $\mathcal{C}$-uniform $\mathcal{C}$-subgroup.

Proof. Temporarily suppose that it is not so. There exist nonzero $\mathcal{C}$-subgroups $K_{1}, J_{1} \subseteq K$ with $K_{1} \cap J_{1}=0$, then there exist nonzero $\mathcal{C}$-subgroups $K_{2}, J_{2} \subseteq K_{1}$ with $K_{2} \cap J_{2}=0$, and ... so on. Now $\left\{J_{i} \mid i \geq 1\right\}$ is an infinite independent set of nonzero $\mathcal{C}$-subgroups contained in $K$ which is a contradiction.

Lemma 2.4. Let $M$ be a $\mathcal{G}$-intersection $\mathcal{G}$-semisimple additive group. If $\star$ is a map such that

(1) For any $\mathcal{G}$-subgroup $K, K \cap K^{\star}=0$.

(2) For any $\mathcal{G}$-subgroups $J$ and $K, K \cap J=0$ implies $J \subseteq K^{\star}$. 
Then, for every $\mathcal{G}$-subgroup $K$ we have $\Sigma\left\langle\mathcal{G}^{\mathrm{mn}} \nsubseteq K\right\rangle=K^{\star}, \Sigma\left\langle\mathcal{G}^{\mathrm{mn}} \subseteq K\right\rangle=K$, $M=K \oplus K^{\star}$ and $K^{\star \star}=K$. Also $\star$ is a $\mathcal{G}$-organizer map.

Proof. For each $J \in\left\langle\mathcal{G}^{\mathrm{mn}}: M\right\rangle$, if $J \nsubseteq K$, then $J \cap K=0$, implying $J \subseteq K^{\star}$. Thus, $\Sigma\left\langle\mathcal{G}^{\mathrm{mn}} \nsubseteq \subseteq K\right\rangle \subseteq K^{\star}$. On the other hand, $\Sigma\left\langle\mathcal{G}^{\mathrm{mn}} \subseteq K\right\rangle \subseteq K$. Therefore, $\Sigma\left\langle\mathcal{G}^{\mathrm{mn}} \not \subset K\right\rangle=K^{\star}, \Sigma\left\langle\mathcal{G}^{\mathrm{mn}} \subseteq K\right\rangle=K$ and $M=K \oplus K^{\star}$. It is clear that $K \subseteq K^{\star \star}$. On the other hand, $K^{\star} \cap K^{\star \star}=0$. Thus, $K^{\star \star}=K$.

Lemma 2.5. Let $M$ be an additive group and $\star$ be a $\mathcal{G}$-organizer map.

(1) For any $\mathcal{G}$-subgroup $K, K$ is $\mathcal{G}$-summand if and only if $M=K \oplus K^{\star}$.

(2) If $M \in\langle\mathcal{G}: M\rangle$, then $K \in\left\langle\mathcal{G}^{\mathrm{mn}}: M\right\rangle$ if and only if $K^{\star} \in\left\langle\mathcal{G}^{\mathrm{mx}}: M\right\rangle$.

Proof. $(1 \Rightarrow)$ There exists a $\mathcal{G}$-subgroup $J$ with $M=K \oplus J$, then $J \subseteq K^{\star}$, implying $J=K^{\star}$.

(2) Straightforward.

Lemma 2.6. Let $M$ be a $\mathcal{G}$-intersection (generalized $\mathcal{G}$-intersection) additive group and $\star$ be a $\mathcal{G}$-organizer map. For any finite set (any set) $A$ of $\mathcal{G}$-subgroups,

(1) If $\Sigma(A)$ is a $\mathcal{G}$-subgroup, then $(\Sigma(A))^{\star}=\operatorname{Int}\left\{I^{\star} \mid I \in A\right\}$.

(2) $A=\left\langle\mathcal{G}^{\mathrm{mn}} \subseteq\left(\operatorname{Int}\left\{I^{\star} \mid I \in A\right\}\right)^{\star}\right\rangle$.

Proof. (1) Set $L=\operatorname{Int}\left\{I^{\star} \mid I \in A\right\}$. For any $I \in A$, have $I \subseteq \Sigma(A)$, so $(\Sigma(A))^{\star} \subseteq$ $I^{\star}$. Thus $(\Sigma(A))^{\star} \subseteq L$. On the other hand, for any $J \in A, J \cap L=0$, implying $J \subseteq L^{\star}$. Thus, $\Sigma(A) \subseteq L^{\star}$, implying $L \subseteq(\Sigma(A))^{\star}$.

(2) For each $J \in A, L \cap J=0$, implying $J \subseteq L^{\star}$. Thus, $A \subseteq\left\langle\mathcal{G}^{\mathrm{mn}} \subseteq L^{\star}\right\rangle$. Also for each $I \in A$ and $J \in\left\langle\mathcal{G}^{\mathrm{mn}}: M\right\rangle$ with $J \notin A, I \cap J=0$, implying $J \subseteq I^{\star}$. So, for each $J \in\left\langle\mathcal{G}^{\mathrm{mn}}: M\right\rangle$ with $J \notin A, J \subseteq L$, implying $J \notin\left\langle\mathcal{G}^{\mathrm{mn}} \subseteq L^{\star}\right\rangle$. Thus, $A=\left\langle\mathcal{G}^{\mathrm{mn}} \subseteq L^{\star}\right\rangle$.

Lemma 2.7. Let $M$ be a $\mathcal{G}$-intersection additive group and $\star$ be a $\mathcal{G}$-organizer map. If $I$ is a $\mathcal{G}$-summand $\mathcal{G}$-subgroup, then for any $\mathcal{G}$-subgroup $J$ we have

(1) $(I \cap J)^{\star}=I^{\star}+J^{\star}$.

(2) $I+J$ is a $\mathcal{G}$-subgroup.

(3) $(I+J)^{\star}=I^{\star} \cap J^{\star}$.

(4) $J=(I \cap J) \oplus\left(I^{\star} \cap J\right)$.

Proof. (1) $M=I \oplus I^{\star}$ by Lemma 2.5. Since $I^{\star} \subseteq(I \cap J)^{\star},(I \cap J)^{\star}=I^{\star} \oplus I \cap(I \cap J)^{\star}$. On the other hand, $(I \cap J) \cap(I \cap J)^{\star}=0$, implying $I \cap(I \cap J)^{\star} \subseteq J^{\star}$. Thus $(I \cap J)^{\star} \subseteq I^{\star}+J^{\star}$. Finally we have $I^{\star}, J^{\star} \subseteq(I \cap J)^{\star}$, implying $I^{\star}+J^{\star} \subseteq(I \cap J)^{\star}$. (2) and (3) $I^{\star}$ is also a $\mathcal{G}$-summand by Lemma 2.5. So, $\left(I^{\star} \cap J^{\star}\right)^{\star}=I^{\star \star}+J^{\star \star}=I+J$ 
by (1). Thus, $I+J$ is a $\mathcal{G}$-subgroup, also $(I+J)^{\star}=\left(I^{\star} \cap J^{\star}\right)^{\star \star}=I^{\star} \cap J^{\star}$.

(4) As we showed in proof of $(1), I \cap(I \cap J)^{\star} \subseteq J^{\star}$, so

$$
J=J^{\star \star} \subseteq\left(I \cap(I \cap J)^{\star}\right)^{\star}=I^{\star}+(I \cap J)^{\star \star}=I^{\star}+I \cap J
$$

implying $J=\left(I^{\star}+I \cap J\right) \cap J=I^{\star} \cap J+I \cap J$.

Lemma 2.8. Let $M$ be a $\mathcal{G}$-intersection and $\mathcal{G}$-organized additive group.

(1) $\left\langle\mathcal{G}^{\mathrm{mn}}: M\right\rangle$ is an independent set.

(2) If $M \in\langle\mathcal{G}: M\rangle$ and $\operatorname{Int}\left\langle\mathcal{G}^{\mathrm{mx}}: M\right\rangle=0$, then $M$ is $\mathcal{G}$-mini.

(3) For any nonzero $\mathcal{G}$-subgroup $K, K$ is $\mathcal{G}$-indecomposable and $\mathcal{G}$-summand if and only if $K \in\left\langle\left(\mathcal{G} \cap \mathcal{G}^{\oplus}\right)^{\mathrm{mn}}: M\right\rangle$.

(4) If $M \in\langle\mathcal{G}: M\rangle$, then for any set $A$ and $B$ of maximal $\mathcal{G}$-subgroups, $A \subseteq B$ if and only if $\operatorname{Int}(B) \subseteq \operatorname{Int}(A)$.

(5) $A \mathcal{G}$-subgroup is $\mathcal{G}$-uniform if and only if it is a minimal $\mathcal{G}$-subgroup.

Proof. (1) There exists a $\mathcal{G}$-organizer map $\star$. Let $J \in\left\langle\mathcal{G}^{\mathrm{mn}}: M\right\rangle$. For each $J \neq I \in\left\langle\mathcal{G}^{\mathrm{mn}}: M\right\rangle, I \cap J=0$, implying $I \subseteq J^{\star}$. Thus $J \cap \Sigma\left(\left\langle\mathcal{G}^{\mathrm{mn}}: M\right\rangle-\{J\}\right)=0$.

(2) Let $K$ be a $\mathcal{G}$-subgroup containing no minimal $\mathcal{G}$-subgroup. For each maximal $\mathcal{G}$-subgroup $J$, we have $K \cap J^{\star}=0$ by Lemma 2.5, implying $K \subseteq J$. Thus $K \subseteq$ $\operatorname{Int}\left\langle\mathcal{G}^{\mathrm{mx}}: M\right\rangle$, implying $K=0$.

$(3 \Rightarrow)$ Let $L$ be a nonzero $\mathcal{G}$-summand $\mathcal{G}$-subgroup contained in $K$. We have $M=$ $L \oplus L^{\star}$ by Lemma 2.5 , so $K=L \oplus\left(L^{\star} \cap K\right)$, thus $L^{\star} \cap K=0$, implying $L=K$.

$(3 \Leftarrow)$ Let $L$ and $N$ be $\mathcal{G}$-subgroups with $K=L \oplus N$ and $N \neq 0$. Then, $M=$ $L \oplus N \oplus K^{\star}$ by Lemma 2.5. On the other hand, $K^{\star}, L \subseteq N^{\star}$, which implies $M=N \oplus N^{\star}$. Thus, $N$ is a $\mathcal{G}$-summand $\mathcal{G}$-subgroup, so $L=0$, implying $N=K$.

$(4 \Leftarrow)$ Temporarily suppose that $A \nsubseteq B$. Consider $J \in A$ such that $J \notin B$. For every $I \in B$ we have $J^{\star} \cap I^{\star}=0$, implying $J^{\star} \subseteq I$. Thus, $J^{\star} \subseteq \operatorname{Int}(B) \subseteq \operatorname{Int}(A) \subseteq J$ which is a contradiction.

$(5 \Rightarrow)$ Let $K$ be a $\mathcal{G}$-uniform $\mathcal{G}$-subgroup. Now let $I$ be a nonzero $\mathcal{G}$-subgroup contained in $K . I^{\star} \cap K$ is a $\mathcal{G}$-subgroup contained in $K$ and $I \cap\left(I^{\star} \cap K\right)=0$, so $I^{\star} \cap K=0$, implying $K \subseteq I^{\star \star}=I$. Thus $I=K$.

Lemma 2.9. Let $M$ be a $\mathcal{G}$-intersection, $\mathcal{G}$-organized and $\mathcal{G}$-semisimple additive group.

(1) Every $\mathcal{G}$-subgroup $K$ is $\mathcal{G}$-summand and $M=K \oplus K^{\star}$.

(2) Every $\mathcal{G}$-subgroup is a sum of minimal $\mathcal{G}$-subgroups.

(3) $M \in\langle\mathcal{G}: M\rangle$ and $M$ is $\mathcal{G}$-mini.

(4) The sum of any finite set of minimal $\mathcal{G}$-subgroups is a $\mathcal{G}$-subgroup. 
$(5)\left\langle\left(\mathcal{G} \cap \mathcal{G}^{\oplus}\right)^{\mathrm{mn}}: M\right\rangle$ is the set of nonzero $\mathcal{G}$-indecomposable $\mathcal{G}$-subgroups.

Proof. (1) and (2) There exists a $\mathcal{G}$-organizer map $\star$. Applying Lemma 2.4 completes the proof.

(3) Let $K$ be a $\mathcal{G}$-subgroup containing no minimal $\mathcal{G}$-subgroup. For each minimal $\mathcal{G}$-subgroup $J$ we have $K \cap J=0$, implying $J \subseteq K^{\star}$. Thus $\Sigma\left\langle\mathcal{G}^{\mathrm{mx}}: M\right\rangle \subseteq K^{\star}$, so $K^{\star}=M$, implying $K=0$. Also we have $M=\Sigma\left\langle\mathcal{G}^{\mathrm{mn}}: M\right\rangle=0^{\star}$ by (1).

(4) Let $A$ be a finite set of minimal $\mathcal{G}$-subgroups. Set $L=\operatorname{Int}\left\{I^{\star} \mid I \in A\right\}$. $A=\left\langle\mathcal{G}^{\mathrm{mn}} \subseteq L^{\star}\right\rangle$ by Lemma 2.6 , implying $\Sigma(A)=L^{\star}$ by $(1)$.

(5) Follows from Lemma 2.8 and (1).

Lemma 2.10. Let $M$ be a generalized $\mathcal{G}$-intersection (or $\mathcal{G}$-intersection and $\left\langle\mathcal{G}^{\mathrm{mx}}\right.$ : $M\rangle$ is finite), $\mathcal{G}$-mini and $\mathcal{G}$-organized additive group with $M \in\langle\mathcal{G}: M\rangle$.

(1) For every $\mathcal{G}$-subgroup $K, \operatorname{Int}\left\langle\mathcal{G}^{\mathrm{mx}} \supseteq K\right\rangle=K$.

(2) $\operatorname{Int}\left\langle\mathcal{G}^{\mathrm{mx}}: M\right\rangle=0$.

Proof. (1) There exists a $\mathcal{G}$-organizer map $\star$. Set $L=\operatorname{Int}\left\{I^{\star} \mid I \in\left\langle\mathcal{G}^{\mathrm{mx}} \nsubseteq K\right\rangle\right\}$. For each $I \in\left\langle\mathcal{G}^{\mathrm{mx}} \nsubseteq K\right\rangle$ we have $I \cap K=0$, implying $K \subseteq I^{\star}$. Thus, $K \subseteq L$. It is enough to show that $K^{\star} \cap L=0$. Temporarily suppose that it is not so. $K^{\star} \cap L$ contains a minimal $\mathcal{G}$-subgroup $J$. Then, $I \nsubseteq K$, implying $J \subseteq L \subseteq J^{\star}$ which is a contradiction.

(2) We have $M^{\star}=0$, so 0 is a $\mathcal{G}$-subgroup. Applying (1) completes the proof .

Lemma 2.11. Let $M$ be a generalized $\mathcal{G}$-intersection and $\mathcal{G}$-organized additive group. If $M$ is $\mathcal{G}$-semisimple, then $M$ is a generalized $\mathcal{G}$-sum.

Proof. It is enough to show that for every $A \subseteq\left\langle\mathcal{G}^{\mathrm{mn}}: M\right\rangle, \Sigma(A)$ is a $\mathcal{G}$-subgroup by Lemma 2.9. Set $L=\operatorname{Int}\left\{I^{\star} \mid I \in A\right\} . A=\left\langle\mathcal{G}^{\mathrm{mn}} \subseteq L^{\star}\right\rangle$ by Lemma 2.6, implying $\Sigma(A)=L^{\star}$ by Lemma 2.9 .

Lemma 2.12. Let $M$ be a $\mathcal{G}$-intersection and $\mathcal{G}$-organized additive group. If every $\mathcal{G}$-subgroup is $\mathcal{G}$-summand, then $M$ is $\mathcal{G}$-sum.

Proof. Follows from Lemma 2.7.

Proposition 2.13. Let $M$ be a $\mathcal{G}$-intersection and $\mathcal{G}$-organized additive group with $M \in\langle\mathcal{G}: M\rangle$. Then the following are equivalent.

(1) $\langle\mathcal{G}: M\rangle$ is finite.

(2) $\left\langle\mathcal{G}^{\mathrm{mn}}: M\right\rangle$ is finite and $M$ is $\mathcal{G}$-mini.

(3) $M$ is $\mathcal{G}$-ind.finite.

(4) $M$ is $\mathcal{G}$-Artinian. 
(5) $M$ is $\mathcal{G}$-Noetherian.

(6) $\left\langle\mathcal{G}^{\mathrm{mx}}: M\right\rangle$ is finite and $\operatorname{Int}\left\langle\mathcal{G}^{\mathrm{mx}}: M\right\rangle=0$.

(7) Every $\mathcal{G}$-subgroup is the intersection of a finite set of maximal $\mathcal{G}$-subgroups.

In this case, $M$ is generalized $\mathcal{G}$-intersection, and if every $\mathcal{G}$-subgroup is a $\mathcal{G}$ summand, then $M$ is $\mathcal{G}$-semisimple.

Proof. $(2) \Rightarrow(7)$ Follows from Lemma 2.10.

$(7) \Rightarrow(6)$ There exists a finite set $A$ of maximal $\mathcal{G}$-subgroups such that $\operatorname{Int}(A)=0$. Thus $\operatorname{Int}(A)=\operatorname{Int}\left\langle\mathcal{G}^{\mathrm{mx}}: M\right\rangle$, implying $A=\left\langle\mathcal{G}^{\mathrm{mx}}: M\right\rangle$ by Lemma 2.8. Consequently, $\left\langle\mathcal{G}^{\mathrm{mx}}: M\right\rangle$ is finite, also $\operatorname{Int}\left\langle\mathcal{G}^{\mathrm{mx}}: M\right\rangle=0$.

$(6) \Rightarrow(2)$ Follows from Lemma 2.8.

(6) and (7) $\Rightarrow(1)$ It is obvious.

$(1) \Rightarrow(3)$ It is obvious.

$(3) \Rightarrow(2)$ Follows from Lemma 2.3 and Lemma 2.8.

$(5) \Rightarrow(3)$ Temporarily suppose that there exists an infinite independent set of nonzero $\mathcal{G}$-subgroups. Then there exists an infinite independent set $\left\{J_{i} \mid i \geq 1\right\}$ of nonzero $\mathcal{G}$-subgroups. Set $K_{n}=\left(\cap_{i=1}^{n}\left(J_{i}\right)^{\star}\right)^{\star}$. $K_{n}$ is a $\mathcal{G}$-subgroups. Also, $J_{n+1} \cap K_{n}=0$ and $J_{n+1} \subseteq K_{n+1}$. Thus $K_{n} \subset K_{n+1}$, which is a contradiction.

$(4) \Leftrightarrow(5)$ It is obvious.

$(1) \Rightarrow(5)$ It is obvious.

Finally $M$ is generalized $\mathcal{G}$-intersection by (1).

Now suppose that every $\mathcal{G}$-subgroup is a $\mathcal{G}$-summand. If $M \neq \Sigma\left\langle\mathcal{G}^{\mathrm{mn}}: M\right\rangle$, then $\left(\Sigma\left\langle\mathcal{G}^{\mathrm{mn}}: M\right\rangle\right)^{\star}$ contains a minimal $\mathcal{G}$-subgroup by $(2)$ which is a contradiction.

Lemma 2.14. Let $M$ be a $\mathcal{G}$-intersection additive group and $\star$ be a $\mathcal{G}$-organizer map. Consider $\mathcal{P}$ as the class of $\mathcal{G}$-summand $\mathcal{G}$-subgroups. Then $\star$ is a $\mathcal{P}$-organizer map.

Proof. Let $K$ be a $\mathcal{P}$-subgroup. It follows from Lemma 2.5 that $K^{\star}$ is also a $\mathcal{P}$-subgroup.

Proposition 2.15. Let $M$ be a $\mathcal{G}$-intersection and $\mathcal{G}$-organized additive group. Consider $\mathcal{P}$ as the class of $\mathcal{G}$-summand $\mathcal{G}$-subgroups. Then

(1) $M$ is $\mathcal{P}$-organized.

(2) $M$ is $\mathcal{P}$-intersection.

(3) $M$ is $\mathcal{G}$-sum.

(4) Every $\mathcal{P}$-subgroup is a $\mathcal{P}$-summand.

(5) $\left\langle\mathcal{P}^{\mathrm{mn}}: M\right\rangle$ is an independent set. 
Proof. (1) There exists a $\mathcal{G}$-organizer map $\star$. Then, $\star$ is a $\mathcal{P}$-organizer map by Lemma 2.14.

(2) Let $I$ and $J$ be $\mathcal{P}$-subgroups. By Lemma 2.7,

$$
M=J \oplus J^{\star}=(I \cap J)+\left(I^{\star} \cap J\right)+J^{\star} \subseteq(I \cap J)+I^{\star}+J^{\star}=(I \cap J)+(I \cap J)^{\star} .
$$

(3) Follows from Lemma 2.12 and (4).

(4) Let $K$ be a $\mathcal{P}$-subgroup. $M=K \oplus K^{\star}$ by Lemma 2.5. On the other hand, $K^{\star}$ is a $\mathcal{P}$-subgroup by (1).

(5) Follows from Lemma 2.8.

Proposition 2.16. Let $M$ be a $\mathcal{G}$-intersection and $\mathcal{G}$-organized additive group with $M \in\langle\mathcal{G}: M\rangle$. Consider $\mathcal{P}$ as the class of $\mathcal{G}$-summand $\mathcal{G}$-subgroups. If $M$ is either $\mathcal{G}$-ind.finite, or $\mathcal{G}$-Artinian or $\mathcal{G}$-Noetherian, then $M$ is generalized $\mathcal{P}$-intersection, $\mathcal{P}$-semisimple and $\mathcal{P}$-organized, also $\langle\mathcal{P}: M\rangle$ is finite.

Proof. $M$ is respectively either, $\mathcal{P}$-ind.finite or $\mathcal{P}$-Artinian or $\mathcal{P}$-Noetherian. Also it is clear that $M \in\langle\mathcal{P}: M\rangle$. Applying Proposition 2.13 completes the proof.

Lemma 2.17. Let $M$ be a $\mathcal{G}$-organized additive group with $M \in\langle\mathcal{G}: M\rangle$. If $M$ is $\mathcal{G}$-d.sum, then any $\mathcal{G}$-subgroup is $\mathcal{G}$-summand.

Proof. Let $K$ be a $\mathcal{G}$-subgroup. Set $L=\left(K \oplus K^{\star}\right)^{\star}$. Then, $\left(K \oplus K^{\star}\right) \cap L=0$, so $K \cap L=0$, thus $L \subseteq K^{\star}$, implying $L=0$. Thus, $M=L^{\star}=K \oplus K^{\star}$.

Proposition 2.18. Let $M$ be a generalized $\mathcal{G}$-intersection, $\mathcal{G}$-semisimple and $\mathcal{G}$ organized additive group.

(1) $M$ is generalized $\mathcal{G}$-sum.

(2) Every $\mathcal{G}$-subgroup is an intersection of maximal $\mathcal{G}$-subgroups.

Proof. (1) It is enough to show that the sum of any set $A$ of minimal $\mathcal{G}$-subgroups is a $\mathcal{G}$-subgroups by Lemma 2.9. Set $L=\operatorname{Int}\left\{I^{\star} \mid I \in A\right\} . A=\left\langle\mathcal{G}^{\mathrm{mn}} \subseteq L^{\star}\right\rangle$ by Lemma 2.6, implying $\Sigma(A)=L^{\star}$ by Lemma 2.9.

(2) Let $K$ be an $\mathcal{G}$-subgroup. Set $A=\left\langle\mathcal{G}^{\mathrm{mn}} \subseteq K^{\star}\right\rangle$. Then, $K^{\star}=\Sigma(A)$ by Lemma 2.9, so $K=\operatorname{Int}\left\{I^{\star} \mid I \in A\right\}$ by Lemma 2.6 and (1). On the other hand, $I^{\star}$ is a maximal $\mathcal{G}$-subgroup for each $I \in A$ by Lemma 2.5 .

Lemma 2.19. Let $M$ be a generalized $\mathcal{G}$-intersection, generalized $\mathcal{G}$-d.sum and $\mathcal{G}$ organized additive group. If $M \in\langle\mathcal{G}: M\rangle$ and $M$ is $\mathcal{G}$-mini, then $M$ is $\mathcal{G}$-semisimple and generalized $\mathcal{G}$-sum. 
Proof. Set $A=\left\langle\mathcal{G}^{\mathrm{mn}}: M\right\rangle$ and $L=\operatorname{Int}\left\{I^{\star} \mid I \in A\right\}$. Then, $(\Sigma(A))^{\star}=L$ by Lemma 2.6 and Lemma 2.8. On the other hand, $L=0$ by Lemma 2.5 and Lemma 2.10 . Thus, $\Sigma(A)=M$.

\section{Block decomposition for modules}

Definition 3.1. Let $R$ be a ring. Two $R$-modules $M$ and $N$ are said to be block orthogonal if $\operatorname{ann}_{N}\left(\operatorname{ann}_{R}(M)\right)=0$ and $\operatorname{ann}_{M}\left(\operatorname{ann}_{R}(N)\right)=0$.

Definition 3.2. Let $R$ be a ring and $M$ be an $R$-module.

(1) For every $P \subseteq M, \operatorname{ann}_{M}\left(\operatorname{ann}_{R}(M / P)\right)$ is denoted by $P^{\circ}$.

(2) $P \subseteq M$ is called an annihilator if $\operatorname{ann}_{M}\left(\operatorname{ann}_{R}(P)\right)=P$.

(3) $M$ is said to be cofaithful if $R$ is $M$-faithful. It should be noted that the use of the term "cofaithful" hear is not the same as the usage in [1].

(4) $M$ is said to be block indecomposable if for any block orthogonal $R$-modules $N$ and $P, M \cong N \times P$ implies either $N=0$ or $P=0$.

(5) If there exist pairwise block orthogonal block indecomposable modules $M_{1}$, $M_{2}, \ldots, M_{n}$ such that $M \cong \prod_{i=1}^{n} M_{i}$, then we say that $M$ is block decomposable.

Also, in the category of modules, the class of annihilators is denoted by $\mathbb{A}$, the class of submodules is denoted by $\mathbb{M}$, the class of annihilator submodules is denoted by $\mathbb{A M}$, and the class of submodules $P$ for which $P \cap P^{\circ}=0$ and $P^{\circ \circ}=P$ is denoted by $\mathbb{C}$.

It is obvious that for any $P \subseteq M, P^{\circ}$ is an annihilator submodule, so $P^{\circ \circ}$ is also an annihilator submodule.

Lemma 3.3. Let $R$ be a ring and $M$ be an $R$-module.

(1) For any subgroup $K \subseteq M$ and any submodule $J, K \cap J=0$ implies $J \subseteq K^{\circ}$.

(2) For any submodules $I$ and $J, I \cap I^{\circ}=0$ and $J \cap J^{\circ}=0$ implies $(I \cap J) \cap$ $(I \cap J)^{\circ}=0$.

(3) For any submodules $I$ and $J$ with $I \cap I^{\circ}=0$ and $J \cap J^{\circ}=0, I \cap J=0$ implies that $I$ and $J$ are block orthogonal.

Proof. (1) Just for the calculations, we may assume that $M$ is a right $R$-module.

We have $J \operatorname{ann}_{R}(M / K) \subseteq K \cap J=0$, so $J \subseteq \operatorname{ann}_{M}\left(\operatorname{ann}_{R}(M / K)\right)=K^{\circ}$.

(2) Set $K=(I \cap J) \cap(I \cap J)^{\circ}$. We have $K(I \cap J: M)=0$. On the other hand,

$$
(I: M)(J: M) \subseteq(I: M) \cap(J: M)=(I \cap J: M) .
$$


So $K(I: M)(J: M)=0$, thus $K(J: M) \subseteq I^{\circ} \cap I=0$, implying $K \subseteq J^{\circ} \cap J=0$.

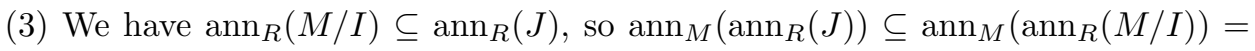
$I^{\circ}$, implying $\operatorname{ann}_{I}\left(\operatorname{ann}_{R}(J)\right) \subseteq I \cap I^{\circ}=0$. Similarly, $\operatorname{ann}_{J}\left(\operatorname{ann}_{R}(I)\right)=0$.

Lemma 3.4. Let $R$ be a ring and $M$ be an $R$-module.

(1) Any two annihilator submodules with zero intersection are block orthogonal.

(2) For any block orthogonal submodules $K$ and $J$ with $K \cap J=0$, if $K \oplus J$ is an annihilator, then $K$ and $J$ are annihilators.

(3) Any submodule which is a block indecomposable module, is $\mathbb{A M}$-indecomposable.

(4) Any $\mathbb{A M}$-indecomposable annihilator submodule is a block indecomposable module.

Proof. (1) Let $K$ and $J$ be annihilator submodules with $K \cap J=0$. Then

$$
\operatorname{ann}_{J}\left(\operatorname{ann}_{R}(K)\right)=J \cap \operatorname{ann}_{M}\left(\operatorname{ann}_{R}(K)\right)=J \cap K=0 .
$$

Similarly, $\operatorname{ann}_{K}\left(\operatorname{ann}_{R}(J)\right)=0$.

(2) Set $N=K \oplus J$. We have $K \subseteq \operatorname{ann}_{M}\left(\operatorname{ann}_{R}(K)\right) \subseteq \operatorname{ann}_{M}\left(\operatorname{ann}_{R}(N)\right)=N$ and $J \cap \operatorname{ann}_{M}\left(\operatorname{ann}_{R}(K)\right)=\operatorname{ann}_{J}\left(\operatorname{ann}_{R}(K)\right)=0$. Thus, $\operatorname{ann}_{M}\left(\operatorname{ann}_{R}(K)\right)=K$. Similarly, $\operatorname{ann}_{M}\left(\operatorname{ann}_{R}(J)\right)=J$.

(3) Let $N$ be a submodule which is a block indecomposable module. Let $K$ and $J$ be annihilator submodules with $N=K \oplus J . K$ and $J$ are block orthogonal modules by (1), also $N \cong K \times J$. Thus either $K=0$ or $J=0$.

(4) Let $N$ be an $\mathbb{A} \mathbb{M}$-indecomposable annihilator submodule. Now let $A$ and $B$ be block orthogonal modules and $N \cong A \times B$. There exist submodules $K$ and $J$ that are block orthogonal modules, $K \cong A, J \cong B$ and $N=K \oplus J . K$ and $J$ are annihilator submodules by (2). Thus $K=0$ or $J=0$ implying $A=0$ or $B=0$.

Lemma 3.5. Let $R$ be a ring and $M$ be an $R$-module. For any submodules $K$ and $J$ with $M=K \oplus J, K$ and $J$ are annihilators if and only if $K$ and $J$ are block orthogonal. In this case

(1) $K^{\circ}=J$ and $J^{\circ}=K$.

(2) $K$ and $J$ are $\mathbb{C}$-summand $\mathbb{C}$-submodules.

Proof. The first statement follows from Lemma 3.4.

(1) $\operatorname{ann}_{R}(K)+\operatorname{ann}_{R}(J)$ is $M$-faithful because

$$
\operatorname{ann}_{M}\left(\operatorname{ann}_{R}(K)+\operatorname{ann}_{R}(J)\right)=\operatorname{ann}_{M}\left(\operatorname{ann}_{R}(K)\right) \cap \operatorname{ann}_{M}\left(\operatorname{ann}_{R}(J)\right)=K \cap J=0 .
$$


On the other hand, $(K: M)=\operatorname{ann}_{R}(J)$ and $\left(K \cap K^{\circ}\right)\left(\operatorname{ann}_{R}(K)+(K: M)\right)=0$. So, $K \cap K^{\circ}=0$. Furthermore $J \subseteq K^{\circ}$ by Lemma 3.3. Thus, $K^{\circ}=J$.

(2) Follows from (1).

Lemma 3.6. Let $R$ be a ring and $M_{1}, M_{2}, \ldots, M_{n}$ and $N$ be $R$-modules. If the pairs, $N, M_{j}$, are block orthogonal for each $1 \leq j \leq n$, then $N$ and $\prod_{i=1}^{n} M_{i}$ are block orthogonal.

Proof. Set $I=\operatorname{ann}_{R}(N)$ and $M=\prod_{i=1}^{n} M_{i}$. Let $m \in M$ and $m I=0$. There exist $m_{i} \in M_{i}$ such that $m=\left(m_{1}, m_{2}, \cdots, m_{n}\right)$. For each $1 \leq j \leq n$, we have $m_{j} I=0$, implying $m_{j}=0$. So $m=0$. Thus $\operatorname{ann}_{M}\left(\operatorname{ann}_{R}(N)\right)=0$. Now set $I_{j}=\operatorname{ann}_{R}\left(M_{j}\right)$ for each $1 \leq j \leq n$. $I_{j}$ is $N$-faithful for each $1 \leq j \leq n$, so $I_{1} I_{2} \cdots I_{n}$ is $N$-faithful, thus $\cap_{i=1}^{n} I_{i}$ is $N$-faithful. Therefore $\operatorname{ann}_{N}\left(\operatorname{ann}_{R}(M)\right)=0$.

Proposition 3.7. Let $R$ be a ring and $M$ be an R-module. Every $\mathbb{A M}$-summand annihilator submodule $K$ is a $\mathbb{C}$-summand $\mathbb{C}$-submodule, $M=K \oplus K^{\circ}$ and $K$ and $K^{\circ}$ are block orthogonal modules. Also the following conditions are equivalent.

(1) $K$ is a block indecomposable module.

(2) $K$ is $\mathbb{C}$-indecomposable.

(3) $K$ is $\mathbb{A M}$-indecomposable.

Proof. There exists an annihilator submodule $J$ with $M=K \oplus J$. Now applying Lemma 3.5 completes the proof.

(2) $\Rightarrow$ (3) Let $I$ and $N$ be annihilator submodules with $K=I \oplus N . I$ and $N$ are block orthogonal by Lemma 3.4. Also, $K$ and $J$ are block orthogonal by Lemma 3.4, so $I$ and $J$ are block orthogonal, thus $I$ and $N \oplus J$ are block orthogonal by Lemma 3.6. Thus $I$ is a $\mathbb{C}$-summand $\mathbb{C}$-submodules by Lemma 3.5. Similarly, $N$ is a $\mathbb{C}$-summand $\mathbb{C}$-submodules. Hence, $I=0$ or $N=0$.

$(3) \Rightarrow(2)$ It is obvious.

(1) $\Leftrightarrow(3)$ Follows from Lemma 3.4.

Notice that Proposition 3.7 shows that the classes $\mathbb{A M} \cap \mathbb{A M} \oplus$ and $\mathbb{C} \cap \mathbb{C}^{\oplus}$ are identical. This means that $\left\langle\left(\mathbb{C} \cap \mathbb{C}^{\oplus}\right)^{\mathrm{mn}}: M\right\rangle=\left\langle\left(\mathbb{A M} \cap \mathbb{A M}^{\oplus}\right)^{\mathrm{mn}}: M\right\rangle$ for every module $M$.

Proposition 3.8. Every module is $\mathbb{C}$-intersection and the map given by $I \longrightarrow I^{\circ}$ is a $\mathbb{C}$-organizer map.

Proof. Let $R$ be a ring and $M$ be an $R$-module. First, let $I$ and $J$ be $\mathbb{C}$-submodules. $(I \cap J) \cap(I \cap J)^{\circ}=0$ by Lemma 3.3, so $(I \cap J) \subseteq(I \cap J)^{\circ \circ}=0$ by Lemma 3.3. 
On the other hand, $(I \cap J)^{\circ \circ} \subseteq I^{\circ \circ}=I$ and similarly, $(I \cap J)^{\circ \circ} \subseteq J$. Thus, $(I \cap J)^{\circ \circ} \subseteq I \cap J$, implying $(I \cap J)^{\circ \circ}=I \cap J$.

Now let $I$ be a $\mathbb{C}$-submodule. We have $\left(I^{\circ}\right)^{\circ \circ}=\left(I^{\circ \circ}\right)^{\circ}=I^{\circ}$, also $\left(I^{\circ}\right)^{\circ} \cap I^{\circ}=$ $I \cap I^{\circ}=0$. So $I^{\circ}$ is also a $\mathbb{C}$-submodule. Thus, the map is well defined. The rest is obvious.

Lemma 3.9. Let $R$ be a ring and $M_{1}, M_{2}, \ldots, M_{n}$ be pairwise block orthogonal R-modules. Set $M=\prod_{i=1}^{n} M_{i}$.

(1) $\iota_{j}\left(M_{j}\right)$ is a $\mathbb{C}$-summand $\mathbb{C}$-submodules for each $1 \leq j \leq n$.

(2) If $M_{j}$ is a block indecomposable module for some $1 \leq j \leq n$, then $\iota_{j}\left(M_{j}\right)$ is a minimal $\mathbb{C}$-summand $\mathbb{C}$-submodule.

Proof. (1) $\Sigma\left\{\iota_{i}\left(M_{i}\right) \mid 1 \leq i \leq n, i \neq j\right\}$ and $\iota_{j}\left(M_{j}\right)$ are block orthogonal by Lemma 3.6 so they are $\mathbb{C}$-summand $\mathbb{C}$-submodules by Lemma 3.5 .

(2) $\iota_{j}\left(M_{j}\right)$ is $\mathbb{C}$-indecomposable by Proposition 3.7. Thus, $\iota_{j}\left(M_{j}\right) \in\left\langle\left(\mathbb{C} \cap \mathbb{C}^{\oplus}\right)^{\mathrm{mn}}\right.$ : $M\rangle$ by Lemma 2.8 .

Proposition 3.10. Let $R$ be a ring and $M_{1}, M_{2}, \ldots, M_{n}$ be pairwise block orthogonal block indecomposable $R$-modules. Set $M=\prod_{i=1}^{n} M_{i}$. Then, $\left\langle\left(\mathbb{A M} \cap \mathbb{A M}^{\oplus}\right)^{\mathrm{mn}}: M\right\rangle=\left\{\iota_{j}\left(M_{j}\right) \mid 1 \leq j \leq n\right\}, M=\Sigma\left\langle\left(\mathbb{A M} \cap \mathbb{A M}^{\oplus}\right)^{\mathrm{mn}}: M\right\rangle$ and $\left\langle\left(\mathbb{A M} \cap \mathbb{A M}^{\oplus}\right)^{\mathrm{mn}}: M\right\rangle$ is finite.

Proof. Lemma 3.9 implies that $\left\{\iota_{j}\left(M_{j}\right) \mid 1 \leq j \leq n\right\} \subseteq\left\langle\left(\mathbb{C} \cap \mathbb{C}^{\oplus}\right)^{\mathrm{mn}}: M\right\rangle$. On the other hand, $M=\Sigma\left\{\iota_{j}\left(M_{j}\right) \mid 1 \leq j \leq n\right\}$ and $\left\langle\left(\mathbb{C} \cap \mathbb{C}^{\oplus}\right)^{\mathrm{mn}}: M\right\rangle$ is an independent set by Proposition 2.15 .

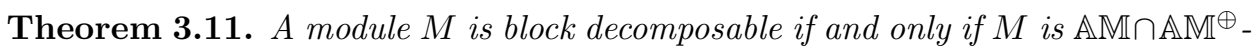
semisimple and $\left\langle\left(\mathbb{A M} \cap \mathbb{A} \mathbb{M}^{\oplus}\right)^{\mathrm{mn}}: M\right\rangle$ is finite. In this case $\bigoplus\left\langle\left(\mathbb{A} \cap \mathbb{A} \mathbb{M}^{\oplus}\right)^{\mathrm{mn}}: M\right\rangle$ is the block decomposition of $M$.

Proof. $(\Rightarrow)$ There exist pairwise block orthogonal block indecomposable modules $M_{1}, M_{2}, \ldots, M_{n}$ such that $M \cong \prod_{i=1}^{n} M_{i}$. We can assume $M=\prod_{i=1}^{n} M_{i}$. Applying Proposition 3.10 completes the proof.

$(\Leftarrow)$ Each $K \in\left\langle\left(\mathbb{A M} \cap \mathbb{A M}^{\oplus}\right)^{\mathrm{mn}}: M\right\rangle$ is $\mathbb{C}$-indecomposable by Lemma 2.8, so is a block indecomposable module by Proposition 3.7 and Proposition 2.15. On the other hand, $\left\langle\left(\mathbb{A M} \cap \mathbb{A M}^{\oplus}\right)^{\mathrm{mn}}: M\right\rangle=\left\{K_{1}, K_{2}, \cdots, K_{n}\right\}$ is an independent set by Lemma 2.5 , so $M \cong \prod_{i=1}^{n} K_{i}$.

Theorem 3.12. Any $\mathbb{A} \mathbb{M}-$-ind.finite, or $\mathbb{A} \mathbb{M}$-Artinian or $\mathbb{A} \mathbb{M}$-Noetherian cofaithful module is block decomposable. 
Proof. Let $M$ be an $\mathbb{A} \mathbb{M}$-ind.finite, or $\mathbb{A M}$-Artinian or $\mathbb{A M}$-Noetherian cofaithful module. Then $M$ is respectively, $\mathbb{C}$-ind.finite, or $\mathbb{C}$-Artinian or $\mathbb{C}$-Noetherian and $M \in\langle\mathbb{C}: M\rangle$. Applying Proposition 2.16 and Theorem 3.11 completes the proof.

The following theorem shows that the block decomposition is unique.

Theorem 3.13. Let $R$ be a ring, $M_{1}, M_{2}, \ldots, M_{n}$ be pairwise block orthogonal block indecomposable $R$-modules, and $N_{1}, N_{2}, \ldots, N_{k}$ be also pairwise block orthogonal block indecomposable $R$-modules. If $\Delta: \prod_{i=1}^{n} M_{i} \longrightarrow \prod_{j=1}^{k} N_{j}$ is an isomorphism, then $k=n$, and after a re indexing, there exist isomorphisms $\Delta_{i}: M_{i} \longrightarrow N_{i}$ for $1 \leq i \leq n$ such that $\Delta=\prod_{i=1}^{n} \Delta_{i}$.

Proof. Set $M=\prod_{i=1}^{n} M_{i}$ and $M=\prod_{j=1}^{k} N_{j} \cdot\left\langle\left(\mathbb{A M} \cap \mathbb{A M}^{\oplus}\right)^{\mathrm{mn}}: M\right\rangle=\left\{\iota_{i}\left(M_{i}\right) \mid\right.$ $1 \leq i \leq n\}$ and $\left\langle\left(\mathbb{A M} \cap \mathbb{A M}^{\oplus}\right)^{\mathrm{mn}}: N\right\rangle=\left\{\iota_{j}\left(N_{j}\right) \mid 1 \leq j \leq k\right\}$ by Proposition 3.10. So $k=n$ and after a re indexing, $\Delta\left(\iota_{i}\left(M_{i}\right)\right)=\iota_{i}\left(N_{i}\right)$ for all $1 \leq i \leq n$. Set $\Delta_{i}: M_{i} \longrightarrow N_{i}$ given by $\Delta_{i}(x)=\pi_{i}(\Delta(\iota(x))$.

\section{Decomposition for rings}

Recall that the class of ideals $I$ for which $I \cap \operatorname{ann}_{l}(I)=0$ and $\operatorname{ann}_{l}\left(\operatorname{ann}_{l}(I)\right)=I$ is denoted by $\mathbb{l} \mathbb{C}$ in $[3]$, and for every left faithful ring $R$, the map $\star:\langle 1 \mathbb{C}: R\rangle \longrightarrow\langle 1 \mathbb{C}: R\rangle$ given by $I^{\star}=\operatorname{ann}_{1}(I)$ is a $\mathbb{l} \mathbb{C}$-organizer map, and $R$ is $1 \mathbb{C}$-intersection and $1 \mathbb{C}$ organized by [3, Lemma 1.6].

Lemma 4.1. Let $R$ be a left faithful ring. If $K$ and $J$ are ideals with $R=K \oplus J$, then $J=\operatorname{ann}_{1}(K)$.

Proof. $\left(K \cap \operatorname{ann}_{l}(K)\right) R=\left(K \cap \operatorname{ann}_{l}(K)\right)(K \oplus J)=0$, so $K \cap \operatorname{ann}_{l}(K)=0$. On the other hand, $J \subseteq \operatorname{ann}_{l}(K)$. Thus $J=\operatorname{ann}_{1}(K)$.

Lemma 4.2. Let $R$ be a left faithful ring.

(1) Any $\mathbb{I}$-summand ideal is a $\mathbb{1} \mathbb{C}$-summand $\mathbb{1} \mathbb{C}$-ideal.

(2) Any $\mathbb{I}$-summand ideal is $\mathbb{1} \mathbb{C}$-indecomposable if and only if it is $\mathbb{I}$-indecomposable.

(3) Any $\mathbb{I}$-summand $\mathbb{I}$-indecomposable ideal is a minimal $\mathbb{1} \mathbb{C}$-summand $\mathbb{1} \mathbb{C}$-ideal.

(4) An $\mathbb{I}$-summand ideal is indecomposable as a ring if and only if it is $\mathbb{I}$ indecomposable.

Proof. (1) Let $K$ be an $\mathbb{I}$-summand ideal. There is an ideal $J$ with $R=K \oplus$ $J$. Then $J=\operatorname{ann}_{l}(K)$ and similarly, $\operatorname{ann}_{l}(J)=K$. So $K \cap \operatorname{ann}_{1}(K)=0$ and $\operatorname{ann}_{1}\left(\operatorname{ann}_{l}(K)\right)=K$. Thus, $K$ is a $\mathbb{l} \mathbb{C}$-summand $\mathbb{l} \mathbb{C}$-ideal.

$(2 \Rightarrow)$ Let $K$ be an $\mathbb{I}$-summand $\mathbb{I} C$-indecomposable ideal. Now let $I$ and $J$ be ideals 
with $K=I \oplus J$, then $I$ and $J$ are $\mathbb{I}$-summand ideals, so they are $\mathbb{I} C$-ideals by (1), thus $I=0$ or $J=0$.

(3) Let $K$ be an $\mathbb{I}$-summand $\mathbb{I}$-indecomposable ideal. $K$ is a $\mathbb{I}$-summand $\mathbb{l}$-ideal by (1). Thus $K \in\left\langle\left(\mathbb{C} \cap 1 \mathbb{C}^{\oplus}\right)^{\mathrm{mn}}: R\right\rangle$ by Lemma 2.8 .

Notice that according to Lemma 4.2, in the category of left faithful rings, the classes $\mathbb{I} \cap \mathbb{I}^{\oplus}, 1 \mathbb{A} \cap \cap 1 \mathbb{A}^{\oplus}$ and $1 \mathbb{C} \cap 1 \mathbb{C}^{\oplus}$ are identical.

Theorem 4.3. A left faithful ring $R$ is decomposable if and only if $R$ is $\mathbb{I} \cap \mathbb{I}^{\oplus}$ semisimple and $\left\langle\left(\mathbb{I} \cap \mathbb{I}^{\oplus}\right)^{\mathrm{mn}}: R\right\rangle$ is finite. In this case $\bigoplus\left\langle\left(\mathbb{I} \cap \mathbb{I}^{\oplus}\right)^{\mathrm{mn}}: R\right\rangle$ is the decomposition of $R$.

Proof. A ring $R$ is decomposable if and only if there exists a finite independent set $A$ of ideals which are indecomposable as a ring such that $R=\Sigma(A)$ and this is called a block decomposition of $R$, [4, page 337] and [2, page 214]. So, $R$ is decomposable if and only if there exists a finite independent set $A$ of $\mathbb{I}$-summand $\mathbb{I}$-indecomposable ideals such that $R=\Sigma(A)$ by Lemma 4.2. Thus, $R$ is decomposable if and only if there exists a finite set $A \subseteq\left\langle\left(\mathbb{I} \cap \mathbb{I}^{\oplus}\right)^{\mathrm{mn}}: R\right\rangle$ such that $R=\Sigma(A)$ by Lemma 4.2 and Proposition 2.16. But the only choice for $A$ is $A=\left\langle\left(\mathbb{I} \cap \mathbb{I}^{\oplus}\right)^{\mathrm{mn}}: R\right\rangle$.

Theorem 4.4. Any $1 \mathbb{A I}$-ind.finite, or $1 \mathbb{A I}$-Artinian or $1 \mathbb{A I}$-Noetherian left faithful ring is decomposable.

Proof. The same as the proof of Theorem 3.12.

The following theorem gives us the uniqueness of the the block decomposition.

Theorem 4.5. Let $R_{1}, R_{2}, \ldots, R_{n}$ and $S_{1}, S_{2}, \ldots, S_{k}$ be indecomposable rings. If $\Delta: \prod_{i=1}^{n} R_{i} \longrightarrow \prod_{j=1}^{k} S_{j}$ is an isomorphism, then $k=n$, and after a re indexing, there exist isomorphisms $\Delta_{i}: R_{i} \longrightarrow S_{i}$ for $1 \leq i \leq n$ such that $\Delta=\prod_{i=1}^{n} \Delta_{i}$.

Proof. The same as the proof of Theorem 3.13.

Theorem 4.6. A left faithful ring $R$ is decomposable if and only if $R_{R}$ is block decomposable. In this case, the block decomposition for $R$ and the block decomposition for $R_{R}$ are identical.

Proof. We have $\left\langle 1 \mathbb{A} \cap \cap 1 \mathbb{A}^{\oplus}: R\right\rangle=\left\langle\mathbb{A M} \cap \mathbb{A M}^{\oplus}: R_{R}\right\rangle$. Applying Theorem 3.11 and Theorem 4.3 completes the proof.

Acknowledgment. The authors would like to thank the referee for the valuable suggestions and comments. 


\section{References}

[1] J. A. Beachy and W. D. Blair, Rings whose faithful left ideals are cofaithful, Pacific J. Math., 58(1) (1975), 1-13.

[2] K. R. Goodearl and R. B. Warfield, Jr., An Introduction to Noncommutative Noetherian Rings, Second Edition, London Mathematical Society Student Texts, 61, Cambridge University Press, Cambridge, 2004.

[3] H. Khabazian, Existence and uniqueness of a certain type of subdirect product, to appear in Casp. J. Math. Sci.

[4] T. Y. Lam, A First Course in Noncommutative Rings, Graduate Texts in Mathematics, 131, Springer-Verlag, New York, 1991.

\section{H. Khabazian}

Department of Mathematical Sciences

Isfahan University of Technology

Isfahan, Iran

e-mail: khabaz@cc.iut.ac.ir 